\title{
Solving the cosmological entropy issue with a Higgs dilaton
}

\author{
David Sloan* \\ Department of Physics, Lancaster University, \\ Lancaster LA1 4YB, United Kingdom \\ and \\ Beecroft Institute of Particle Astrophysics and Cosmology, \\ Department of Physics, University of Oxford, \\ Denys Wilkinson Building, 1 Keble Road, \\ Oxford, OX1 3RH, United Kingdom \\ George Ellis † \\ Mathematics Department, University of Cape Town, \\ Rondebosch, Cape Town 7701, South Africa

\begin{abstract}
Current cosmological models require the universe to be in a very smooth initial state before the onset of inflation, a situation to which Penrose ascribes a vanishingly small probability, leading to his proposal of a Conformal Cyclic Cosmology. We present an alternative paradigm, in which the Higgs plays the role of dilaton and resolves this problem by weakening gravity at very early times, thus providing a form of inflation that is compatible with observations and in which the inflaton is solidly related to tested particle physics.
\end{abstract}

PACS numbers: 04.20.Dw,04.60.Kz,04.60Pp,98.80Qc,04.20Fy

\section{INTRODUCTION}

This paper proposes a unified solution to two issues of major import in current cosmology. First, what is the nature of the inflaton? A major problem at present is a lack of a proposal that is bound in to established particle physics in a solid way. Second, why is the early universe in a very special state that allows inflation to start, when that is a highly improbable situation when one takes the entropy associated with possible primordial black holes into account [35]?

We propose solving both issues simultaneously by using a Higgs dilaton as the inflaton, but coupled in such a way that gravity is essentially turned off in the very early universe. Our purpose in this paper is to show that through suitable couplings the Higgs field can be used to answer both issues. This unifies proposals made by others into a coherent whole that is well worth exploring further.

\footnotetext{
*Electronic address: sloand@lancaster.ac.uk

${ }^{\dagger}$ Electronic address: george.ellis@maths.uct.ac.za
}

\section{A. The nature of the inflaton}

The theory of inflation is a huge success, being able to explain cosmological observations to high precision [1, 2]. However it has a major defect: we have no solid basis for stating what the inflaton is. A great many models have been put forward 33, with some fitting the observations better than others; but most have no sound basis in established physics. The inflaton fields may have their roots in some underlying theory such as string theory or supergravity, be an effective description of a higher order theory of gravity (e.g. Starobinsky inflation [38]), or simply be designed for purpose.

'Designer' inflaton potentials are particularly problematic as they have been shown to be an implementation of Synge's g-method [17]: one can choose from a very broad range of geometric features of a model as desired, and then run the Einstein equations $G_{a b}=\kappa T_{a b}$ from left to right to find the matter content that would give rise to this behaviour, thereby giving an exaat solution of the field equations with the desired properties [39]. In cosmology one can pick any behaviour 
of the scale factor required to produce the observations, and then (subject to a few caveats) find a suitable potential $V(\phi)$ to give rise to the required evolution [18]. As such, ad hoc inflaton models are devoid of meaningful physical content; had we observed any other behaviour of our universe, we could derive a model that would be compatible with those observations to 'explain' them in this way.

The successes of inflationary models are primarily induced by the geometries of the spacetimes they bring about. The spectral tilt is brought about by the slow reduction of the extrinsic curvature, and the solutions to the horizon and flatness problems have their roots in this extended, almost-de Sitter phase. Therefore the question of inflation should not be is there a scalar field that can bring about this behaviour?' to which the answer in any universe is 'yes' (as just explained), but rather 'does known physics produce inflation with this behaviour?' At present most scalar field models are not based on tested, observable physics.

There is however one exception: Higgs inflation [10] is possible 9,25 and fits the observations very well [33]. For reviews see: [7] [37] This is the one and only case in which the inflaton is related to tried and tested physics [20, because the Higgs has indeed been observed at the LHC. Because its properties underlie key features of the standard model of particle physics, if the Higgs were to be the inflaton, one would have one of the most awesome unifications imaginable in physics: the same particle is responsible both for mass at the microscale, and for the dynamics of the very early universe, and hence controls the seeds of structure formation at macro scales. This is therefore a proposal that should be seriously pursued to see if it might work 20]. It is the one possibility for an inflaton solidly tied in to the standard model of particle physics.

In this paper we depart from the aforementioned models in which new scalar fields not known previously are introduced, by using a construction that is in a sense minimal: it only requires known standard model (SM) physics coupled (in a non-minimal way) to gravity hrough a single field that already occurs in the SM.

\section{B. The fine tuned initial state}

The second point is that while it is often stated that inflation solves the flatness and horizon problems in the early universe, that is not in fact the case, as Penrose has pointed out in various writings that are summarised in Chapter 3 of Fashion, Faith, and Fantasy [35. The essential point is that the maximum gravitational entropy of a given amount of matter is attained by collapsing it into a black hole. By contrast, "the Big Bang was an event of extraordinarily low entropy ... the gravitational degrees of freedom were completely suppressed" ([35]:258). Penrose estimates the extraordinary precision that was involved in setting the initial state of the universe as $10^{-10^{123}}$ ([35]:275).

Penrose's argument is simple and persuasive. Consider the space of all possible universes that could have been created by the big bang subject to the condition that they all have the same number of baryons as in our observable universe. The entropy per baryon in the cosmic microwave background has been found to be around $10^{9}$ [41]. However, had the same baryons been packed into stellar mass black holes one would find an entropy per baryon on the order of $10^{20}$, and for supermassive black holes such as the one found at the center of our galaxy this becomes around $10^{26}$. Thus it is apparent that in order to increase the entropy per baryon, baryons should be concentrated in enormous black holes, and to maximize it the entire mass of the universe should be in a single black hole, which would have an approximate entropy of $10^{123}$ which completely dwarfs the observed $10^{89}$. From Boltzmann's law, we know that the entropy of a configuration is the logarithm of the phase-space volume it occupies. Hence if we consider the phase-space of all possible configurations, a universe such as ours occupies a fraction $\mathcal{P}$ of phase space given as the ratio of the exponents of the related entropies;

$$
\mathcal{P}=\frac{e^{S_{1}}}{e^{S_{2}}} \approx \frac{e^{10^{89}}}{e^{10^{123}}} \approx e^{-10^{123}}
$$

Note that in his argument, Penrose points out that in such numbers the difference between $e$ and 10 is insignificant given the enormity of the 
exponents. This argument invokes a uniform measure on phase space; that is assuming the big bang had an equal probability of distributing baryons in all possible configurations. Whilst this may be modified somewhat by various probability measures, to overcome the large factor obtained would require a very special measure.

An objection to Penrose's reasoning along anthropic lines may be raised: If all baryons in the universe are inside black holes then observers such as ourselves would not exist to see them. However, these are easily overcome by a minor perturbation to the reasoning; if we restrict ourselves to conditions under which a galaxy similar to ours formed we still find that the overwhelming majority of such systems (as measured fractionally on phase space as above) would have most of the matter in a super-massive black hole. We similarly note that the overall number of baryons does not play a significant role in the argument itself; a universe with any baryonic content sufficient to produce e.g. our sun will find the black hole configuration entropically favoured.

In order to address questions of probability more fully, one should form a triple consisting of the space of all possible universes, a measure upon that space which assigns a probability of realising any given universe, and a set of observables of interest (in this case the number of baryons in black holes). Since the space of all possible universes remains an open question, this is not possible. The complete space of physically distinct solutions to General Relativity is unknown. However this is not an impediment to Penrose's argument. We know the relative entropy per baryon in the case in which black holes are common and those where they are rare, with the former greatly outweighing the latter. Although we may not know the complete set of solutions, we do know that the set of solutions that look like our universe is vastly outnumbered by those wherein the overwhelming majority of baryons are inside black holes. Thus we should naively expect universes like ours to occur far less frequently than those which are black hole dominated.
This situation is not made clear by standard inflationary studies because they consider only perturbed Robertson-Walker geometries.

An argument due to Kleban - citing a series of studies of inhomogeneous initial conditions for inflation [31 [16] [15] [14] - states that one should consider the deSitter horizon in calculating entropy. Take the space of Schwarzschild-de Sitter space-times and maximize the total horizon area (the sum of the black hole horizon and the de Sitter horizon) holding fixed the cosmological constant. Then this area is maximized by empty de Sitter space, and therefore this should be considered the highest entropy state. We object to this line of reasoning on three grounds: The first is that a de Sitter horizon is not equivalent to a trapping surface. The latter have been shown to obey the laws of thermodynamics with area replacing entropy, whereas the former do not interact with one another. Further, a trapping surface is a locally defined feature of spacetime, whereas there is a deSitter horizon running through every point in de Sitter space. Finally, note that this argument requires the maximization of area subject to having a set cosmological constant but allowing the matter content to vary. This is not the same as addressing the maximal entropy configuration subject to a given baryonic mass in the universe. If we also hold fixed the baryonic mass we see that a homogeneous distribution within the deSitter horizon leads to a smaller total area (and hence entropy) of cosmological horizon than the combined Schwarzschild-de Sitter horizons. The case where matter has formed a black hole will remain static, whereas the homogeneous matter distribution will evolve, its cosmological horizon eventually reaching the end-point of that determined by the dark energy alone. If one is to consider a horizon brought about by a cosmological constant, therefore, the only reasonable way to treat this is as a fixed number which does not affect dynamics.

But additionally, this argument appears to hinge on the proposition that the Universe is at some stage actually de Sitter, which will lead to the Gibbons-Hawking de Sitter horizon properties and associated entropy. However the real 
universe is never de Sitter, and those properties depend on exact symmetries which will not apply. It might possibly asymptote to de Sitter in the far future, or it might not; whether this will happen depends on the nature of dark energy which is unknown. This will in any case not relate to the issue of concern to Penrose as regards entropy of black holes at the start of the universe and the probability of inflation starting. What might possibly be relevant there is a de-Sitter like inflationary stage once it has started: but that again is not de Sitter in the real universe, as is proven by the Planck observations (which show that $r<1$ ). We cannot see how de Sitter can be regarded as a maximal entropy state in contrast to the black hole possibility as discussed above.

Penrose proposes to solve the problem of the initial conditions by invoking a Conformal Cyclic Cosmology ([35]: §4.3). This is a creative idea but the mechanisms involved in realizing such a proposal are not all clear.

\section{Turning off gravity at very high densities}

By contrast, we propose to invoke a mechanism proposed by Greene et al [26]: namely that the strength of the gravitational force dies away at very high energies. In that case the collapse to black holes that causes the problems identified by Penrose will not occur: thermal forces will be able to overcome gravitational forces in the very early universe and prevent collapse to blackholes. The gravitational degrees of freedom will not be suppressed, they will simply not dominate the degrees of freedom of ordinary matter as in the standard case considered by Penrose. Given enough time, thermal processes will result in a uniform state of matter, which will allow the geometry to also be smooth.

There is an issue here as to whether there will be a sufficient time available for such equilibriation to take place; that will not always be the case. However if we assume the universe starts off in a most probable state, that will be a state of maximal entropy, which will indeed be smooth as in the case where gravity is completely switched off. This may be taken as a reasonable initial assumption, expressing the idea, underlying inflationary cosmology, that the starting configuration of the universe (through whatever creation mechanism) is the most probable one.

\section{Putting them together}

Thus we propose to combine these ideas: we will use a Higgs inflaton in a context where gravity is turned off at high densities by the kinds of couplings considered by Greene et al [26. ${ }^{1}$ In physical terms, we make Newton's constant dynamical a la Jordan-Brans-Dicke but with the Higgs playing the role of the coupling. We aim to show this is then a viable cosmological theory that embodies the unifications identified above.

Black hole entropy The BekensteinHawking entropy is determined by the surface area $A$ of a black hole event horizon as measured asymptotically flat spacetime, together with the fundamental constants $c, G$, and $h$, and is given by:

$$
S_{B H}=\frac{c^{3} A}{4 G \hbar}
$$

In the case of a Schwarzschild black hole, $A=16 \pi\left(\frac{G M}{c^{2}}\right)^{2}$ and hence we find that we should expect that the gravitational entropy of black holes is linearly dependent on the strength $G$ of the effective Newton constant. Note that we are thus broadly in keeping with the second law of thermodynamics only if $G$ is increasing.

Naturalness Proposals for Higgs inflation have sometimes been criticized as beung "unnatural", as they require very large values of the coupling parameter between the Higgs field and gravity. When modifying the gravitational Lagrangian from $R$ to $R+\xi \phi^{2} R$ for example, it has

\footnotetext{
${ }^{1}$ A somewhat similar proposal was made by Alexander et al [3 but they did not relate it to the gravitational entropy problem identified by Penrose. In any case the inflaton dynamics involved in that case does not work (Section $\mathrm{VB}$ ).
} 
been claimed that the large value of $\xi$, on the order of $10^{4}$ goes against 'naturalness' [12] (however, it should be noted that this requirement can be brought about from order unity terms [5]). In particular Barbón and Espinosa [6] argue that Higgs inflation requires the field to take values well above the scale at which the effective field theory used is valid. Since the exact theory is not known at these scales, there is no good reason to simply extrapolate the standard model to this point, and therefore the situation is no more natural than that of choosing an inflationary potential. The plateau which is induced in the Einstein frame is sensitive to such corrections, and thus the desirable properties on which Higgs inflation makes it case may not survive a closer analysis. This asymptotic 'shift symmetry' 9] corresponds to scale invariance in the Jordan frame. The naturalness issue comes in the form of a tuning of the ratio between the square of the coupling of Higgs to the Ricci scalar and the large field value form of the potential, which extends to scales well above the cut-off. In the usual Higgs inflation models, this asymptotes to unity and thus the transformed field in the Einstein frame has a plateau (see section V). However this choice may not be justified, and Barbón and Espinosa argue many choices of asymptotic behaviour are possible, some compatible with inflation, some not.

In the current work we allow this coupling to be more general. Using a scalar field toy model, Lerner and McDonald 32 claim that Higgs inflation is natural nonetheless, as the need to introduce new couplings may simply be a failing of the perturbative analysis used in performing calculations, but the underlying physics remains valid. Burgess et al. [13] show the cutoff energy scale appears at the same region in either frame, and note that there the Higgs scattering amplitudes calculated beyond the cutoff scale depend subtly upon the degrees of freedom of the Higgs doublet. This is evidence that the semiclassical approximation has broken down at this scale, and it is therefore not valid to draw conclusions about Higgs inflation coming from energies at or above this level. It is noted by Beruzkov and collaborators 9 that the energy scales at which the effective field theory can be considered valid are dependent on the background energy of the field (Higgs) itself. Thus they are able to show that in the Einstein frame the cutoff during the necessary inflationary phase is above the Planck scale, and thus far higher than the required energy density of the Hubble expansion, therefore the dynamics are well modelled far into the region necessary for agreement with observations. We do not take a position on either side of the arguments presented, but note that Hossenfelder [27] has trenchantly pointed out that such 'naturalness' criteria may be very misleading, and should not in general be taken as hard guidelines for physical theories. In our case we adopt the same position: given the other advantages flowing from the proposed unification, we do not see that any naturalness objection destroys our proposal.

A basis in quantum gravity theory? We do not at this time have any proposal as to how the effective theory we put forward might have a deeper foundation in an underlying more fundamental theory of gravity. This would obviously be desirable. We take our choice to be justified by the unification of different aspects of cosmology achieved, as set out above.

This paper Section II deals with foundations. Section [II considers the case where the field starts off at small values, and Section IV the case where the field starts off at large values. Section $\mathrm{V}$ deals with the effect of frame transformations, and Section VI considers issues that arise.

\section{FOUNDATIONS}

We begin considering a scalar field $\phi$ nonminimally coupled to gravity through some function $F(\phi)$ which multiplies the scalar curvature $R$. Then the action is

$$
S=\int \sqrt{g}\left(\frac{F(\phi)}{6} R+\frac{1}{2} \partial_{a} \phi \partial^{a} \phi-V(\phi)\right)
$$

where $V(\phi)$ is the potential. We have chosen the factor of 6 in the normalisation of the function $F$ to make algebra more convenient when spe- 
cializing this to the case of a Robertson-Walker geometry.

\section{A. Cosmology}

Let us now restrict ourselves to a homogeneous, isotropic spacetime with curvature $k=$ $\pm 1,0$. Thus the partial derivatives are simply time derivatives (up to a choice of lapse). The Ricci scalar in the case of a Robertson-Walker geometry is:

$$
R=6\left(\frac{\ddot{a}}{a}+\frac{\dot{a}^{2}}{a^{2}}+\frac{k}{a^{2}}\right)
$$

where $a(t)$ is the scale factor and $k=\{ \pm 1,0\}$ the normalised spatial curvature. Since the action contains second derivatives of the fields, we integrate by parts and recover (up to boundary terms) a Lagrangian which closely resembles the familiar case of minimally coupled matter, with an extra term arising from the variation of $F$, with $F$ implicitly dependent upon $\phi$ :

$$
\mathcal{L}=a^{3}\left(-F\left(\frac{\dot{a}^{2}}{a^{2}}+\frac{\dot{a} F^{\prime}}{a F} \dot{\phi}-\frac{k}{a^{2}}\right)+\frac{\dot{\phi}}{2}-V\right)
$$

wherein primes denote derivates with respect to the field $\phi$.

The dependence of $F$ on the field $\phi$ leads to modifications of the momenta conjugate to $\phi$ and $a$ from their usual forms. Varying the Lagrangian with respect to the velocities of each of these in turn yields:

$$
\begin{aligned}
& P_{\phi}=a^{3} \dot{\phi}-F^{\prime} a^{2} \dot{a} \\
& P_{a}=-F^{\prime} \dot{\phi} a^{2}+2 F \dot{a} a=-\frac{d}{d t}\left(F a^{2}\right) .
\end{aligned}
$$

Thus we can find the Hamiltonian (the Noether charge associated with time translation), which determines the Friedmann equation from the Einstein-Hilbert action: ${ }^{2}$

$\mathcal{H}=-a^{3} F\left(H^{2}+\frac{F^{\prime}}{F} \dot{\phi} H+\frac{k}{a^{2}}\right)+a^{3}\left(\frac{\dot{\phi}^{2}}{2}+V\right)$

\footnotetext{
${ }^{2}$ The cosmological equations are given in the Appendix.
}

with $\mathcal{H}=0$ as it is a constraint, and wherein we have introduced the standard terminology $H=\dot{a} / a$ for the Hubble parameter. We note that in the case of the reduction $F^{\prime} \rightarrow 0$ this reproduces the usual Friedmann equation, however otherwise we have a new term introduced by the variation of $F$. The extra term breaks the usual relationship between expansion and energy density, thus we should expect to see more interesting dynamics away from stationary points of $F$, as we will have to solve a quadratic equation for $H$. Introducing

$$
\rho=\frac{\dot{\phi}^{2}}{2}+V
$$

in the case of the scalar field (in general $\rho$ represents the energy density of any matter present and minimally coupled to gravity) we see:

$$
H=-\frac{F^{\prime} \dot{\phi}}{2 F} \pm \sqrt{\frac{F^{\prime 2} \dot{\phi}^{2}}{4 F^{2}}+\frac{\rho}{F}-\frac{k}{a^{2}}}
$$

Since the additional term under the square root is positive for $F>0$ such functions would not introduce qualitatively new features (such as bounces in the $k=0,-1$ cases) through the role of $F$. We also retain two branches to cosmological solutions, one expanding $(H>0)$, the other contracting $(H<0)$.

The Euler-Lagrange equations for our system give the dynamics of the fields. The motion of the scalar field is the usual Klein-Gordon equation modified by a gravitational source term proportional to $F^{\prime}$ :

$$
\ddot{\phi}+3 H \dot{\phi}+V^{\prime}=F^{\prime}\left(\dot{H}+2 H^{2}+\frac{k}{a^{2}}\right)
$$

and the usual Raychaudhuri equation is replaced by

$$
\begin{aligned}
2 F \dot{H} & +3 F H^{2}+F \frac{k}{a^{2}}+2 F^{\prime} \dot{\phi} H+F^{\prime} \ddot{\phi}+F^{\prime \prime} \dot{\phi}^{2} \\
& =3\left(V-\frac{\dot{\phi}^{2}}{2}\right) .
\end{aligned}
$$

These equations each reduce to their regular forms in the case $F^{\prime}, F^{\prime \prime} \rightarrow 0$, but have corrections away from such points. We can combine them to find a more informative version of the 
Klein-Gordon equation by eliminating the gravitational source term. Using 2.9 to eliminate terms in $\dot{H}$ and 2.7 to deal with the remainder we arrive at

$$
\ddot{\phi}+3 H \dot{\phi}+\frac{V^{\prime}+\frac{F^{\prime}}{F}\left(\left(1-F^{\prime \prime}\right) \frac{\dot{\phi}^{2}}{2}-2 V\right)}{1+\frac{F^{\prime 2}}{2 F}}=0 .
$$

\section{B. Stationary Points}

An important feature is that this equation reveals that the stationary point $(\ddot{\phi}=\dot{\phi}=0)$ of the scalar field may have shifted - it is no longer necessarily at the minimum of the potential but rather is determined by:

$$
\frac{V^{\prime}}{V}=2 \frac{F^{\prime}}{F}
$$

Here we note that depending of the functional forms of $V$ and $F$ there could be many realizations of the above condition, or none. If we find there to be such a condition away from the minimum of the potential, this appears as a new term in the effective matter energy density of the system; if viewed through the lens of minimally coupled GR, it is an effective cosmological constant. It also potentially has interesting ramifications for reheating.

Let us consider the cases of $V$ having a stable point at $\phi_{s}$ and a global minimum at $\phi_{o}$. Effects during reheating can come around from two possible dynamical events. Since the Higgs will oscillate about a stationary point, $\phi_{s}$ that is not at the minimum of its potential $\left(\phi_{o}\right)$, it may tunnel from this point to close to its global minimum (another stable point). Thus the oscillatory phase in which there is particle production through decay of the Higgs may be interrupted by this sudden transition. Further these two states could introduce different effective couplings - if the interaction Lagrangian is given

$$
\mathcal{L}_{\text {int }}=-g \sigma \phi^{2} \chi^{2}
$$

say then oscillations about $\phi_{s}$ which are insufficient to reach $\phi_{o}$ will produce particles at a different rate than oscillations about $\phi_{o}$. Furthermore, the canonical reheating scenario has an effective, time-averaged equation of state $w_{r e}=0$ corresponding to simple harmonic motion of the inflaton about its minimum. However, in oscillations about this new minimum, a choice of $F$ can significantly alter this giving rise to varied values for $w$ and hence altering the reheating process 34 .

If this does not occur, the minimum will be at the Higgs VEV. In either case, at the stationary point $F$ becomes fixed. Thereafter the system will be described by GR with a fixed Newton constant, whose value is set by the inverse of $\mathrm{F}$ at this stationary point.

\section{SMALL INITIAL FIELD}

We first consider the case where our scalar field begins at small field values, close to the origin. This of course raises issues of naturalness - it would appear that the field would need fine-tuning for this to occur in nature. However, we shall see that there are certain situations in which this can be alleviated. Specifically we take:

$$
\begin{aligned}
& F=A^{2} \exp \left[-\left(\frac{\phi}{S}\right)^{n}\right] \\
& V=\left(\Phi^{2}-\phi^{2}\right)^{2}
\end{aligned}
$$

with $A, S$, and $\Phi$ constants and $n$ a postive integer and will include other minimally coupled matter, labelled by its energy density $\rho$. We will choose $S^{2}<<\Phi^{2}$ so that the dynamics of the Higgs potential is close to that of the standard model when we approach the minimum of the potential.

\section{A. Gaussian $F$}

The first thing to notice is that in the case that $F$ is Gaussian $(\mathrm{n}=2)$ we find that $\phi=0$ becomes a stable minimum of the system. To see this consider the Klein-Gordon equation in this setup. Linearly perturbing $\phi$, to order $\epsilon$ in both $\phi$ and $\dot{\phi}$ we see that:

$$
\ddot{\epsilon}+3 \sqrt{\rho+\Phi^{4}} \dot{\epsilon}+\left(\frac{\rho}{S^{2}}+\frac{4 \Phi^{4}}{S^{2}}-4 \Phi^{2}\right) \epsilon=0
$$


and our condition on $S^{2}$ means that the coefficient of $\epsilon$ is positive. In such a case we could relax our tuning of the system somewhat, as small perturbations about the hilltop in $F$ and $V$ would not lead to run-away dynamics. Any initial condition with $\phi_{0}^{2}<\Phi^{2}-S^{2}$ will tend toward $\phi=0$, and settle there classically. However, this condition holds indefinitely, and as such it would not be possible for the particle to escape from this setup, unless it were to tunnel. That would be the only way to end inflation.

At this point it is useful to note that this system will have five stationary points: $\phi=0, \phi=$ $\pm \Phi$ are stable, $\phi= \pm \sqrt{\Phi^{2}-S^{2}}$ are unstable. To see this consider whether the Klein Gordon equation with $\dot{\phi}=0=\ddot{\phi}$ can be satisfied by solutions to equation 2.11, which in this case implies the conditions for unstable equilibria. Likewise $\phi=\Phi$ is stationary. To see stability, we can expand our solutions to first order in $\phi, \dot{\phi}$ about these points. Setting $\phi=\sqrt{\Phi^{2}-S^{2}}+\epsilon$ we see

$$
\ddot{\epsilon}+3 H \epsilon-\frac{8 H\left(\Phi^{2}-S^{2}\right)}{H+2\left(\Phi^{2}-S^{2}\right)}=0
$$

hence these are unstable equilibria, as the coefficient of $\epsilon$ is negative. Now let us consider the minima of the Higgs potential: setting $\phi=\Phi+\epsilon$ we find

$$
\ddot{\epsilon}+\frac{8 S^{4} \Phi^{2}}{S^{4}+2 \Phi^{2} A^{2} \exp \left[-\Phi^{2} / S^{2}\right]} \epsilon=0
$$

where the coefficient of $\epsilon$ is positive and hence we have a stable equilibrium. At this point the effective Planck mass is $M_{p}^{2}=A^{2} \exp \left[-\Phi^{2} / S^{2}\right]$. Fixing this at this point to match with observations made in the late universe, we see that if the field can move away from the equilibrium point at the origin out to the Higgs minimum by a tunnelling process, the effective Newton constant will have fallen by a factor of $\exp \left[\Phi^{2} / S^{2}\right]$.

In order for the classical motion to no longer move the field towards the hilltop the tunnelling would have to take the particle beyond $\phi=\sqrt{\Phi^{2}-S^{2}}$ - the next (unstable) equilibrium point of the system - and close to the minimum of the potential. From this point onwards the dynamics could reproduce that of slow-roll infla- tion: The slow roll parameters ${ }^{3}$ are $\epsilon_{H}=\frac{-H^{\prime}}{H^{2}}$ and $\eta_{H}=\frac{H^{\prime \prime}}{H^{\prime} H}$, which to first order about this point can be expressed in terms of the field difference from the point, $\delta=\phi-\sqrt{\Phi^{2}-S^{2}}$ :

$$
\begin{aligned}
\epsilon_{H} & \approx \frac{8 A \Phi^{3} \delta}{S^{4} \exp \left[\frac{\Phi^{2}}{2 S^{2}}\right]}=\frac{8 M_{p} \Phi^{3}}{S^{4}} \delta \\
\eta_{H} & \approx \frac{24 A \Phi^{3} \delta}{S^{4} \exp \left[\frac{\Phi^{2}}{2 S^{2}}\right]}=\frac{24 M_{p} \Phi^{3}}{S^{4}} \delta
\end{aligned}
$$

at which points the Hubble parameter takes the value $H=\frac{S^{2}}{A} \exp \left[\frac{\Phi^{2}}{2 S^{2}}\right]=\frac{S^{2}}{M_{p}}$. Thus we see that we have simply replaced one issue of fine-tuning with another; the system would have to tunnel incredibly close to the unstable equilibrium point to begin a slow-roll inflation which was compatible with observations. We therefore turn our attention to other possible choices of $F$.

\section{B. Even powers of $\phi$}

The next logical one is given by setting $n=4$. Unfortunately this, and all higher (even) powers also suffer from having multiple stable minima that are away from the minimum of the Higgs potential. It is, of course, possible to tune such minima so that one of them is compatible with the cosmological constant, however doing so would be essentially no less arbitrary than adding a constant to the potential to recover the same effect. An interesting case in which this occurs due to a more natural mechanism created by the Higgs vacuum being dynamic is presented in [11.

\section{Free choice of $F$}

Let us now consider whether a small initial field choice can ever resolve the problem of gravitational entropy, with the function $F$ kept largely free. In order to have the field begin at small values we require that at least the point

\footnotetext{
${ }^{3}$ We here use the geometric version of the slow-roll condition rather than the potential form, as it is $H$ not $V$ which determines the behaviour of perturbations, and our dynamics is not simply dependent on $V$.
} 
$\phi=0$ be an equilibrium point of the system. For our argument we do not here need to require stability at this point. From the Klein-Gordon equation we see that $\phi=0$ being an equilibrium requires that $F^{\prime}=0$ at this point. Our second requirement is that we want to avoid there being a stationary point of the system for $\phi \in[0, \alpha \Phi]$ for some positive number $\alpha$ so that our cosmology does not enter a deSitter phase that is incompatible with observations. From the current value of the cosmological constant and the potential $V$ we see that this requirement is that $\alpha \approx\left(1-10^{-37}\right)$. Our requirement that there be no stationary points means that equation 2.11 cannot be satisfied on this interval. Hence:

$$
2(\log F)^{\prime}<\left(\log V^{\prime}\right)
$$

SO

$$
\frac{F(0)}{F(\alpha \Phi)}<\sqrt{\frac{V(0)}{V(\alpha \Phi)}}=\frac{\Phi^{2}}{\sqrt{\Lambda}} \approx 10^{37}
$$

Let us summarise this result: If we want to have the effective Newton constant $G$ be a function of the Higgs field such that it does not generate a cosmological constant which is significantly larger than the one currently observed and the field begins its dynamics at the origin, then the maximum ratio of the value of $G$ today to its early value is approximately $10^{37}$. Whilst this is a very large number, it is not enough to overcome the criticisms that Penrose has levelled about the fine-tuning of the initial state: the initial entropy per baryon would be dropped by $10^{37}$, which is not enough to overcome the factor of $10^{123}$ that Penrose calculates (he calculates the probability of being in this state to be $10^{10^{123}}$ and the entropy is the log of this, hence $10^{123}$ ). Starting near the origin appears to fail entirely unless one is willing to have very fine-tuned quantum tunnelling out of such a phase and have $F$ extremely high at the origin.

Note further that this is a very liberal estimate on the upper bound for this ratio; if we were to make the more conservative assumption that the dynamics of the Higgs field was unaffected in a larger region about its minimum we would obtain a much lower bound. For example, insisting that the effective Newton constant is within a few percent of its current value for $\phi>0.99 \Phi$ would restrict this ratio to being in the region of 50 .

\section{In conclusion:}

if we don't want to require tunnelling nor introduce a new cosmological constant this really rules out the small field case as a solution to the entropy problem pointed out by Penrose. A (stable) stationary point of the scalar field away from the minimum of the potential introduces a cosmological constant, and the classical dynamics comes to a rest at this point. We want to rule that out if it would give an effective cosmological constant orders of magnitude bigger than our own today. This means that there's a condition relating the ratio of the potentials today and at the origin, and the ratio of the function $F$ today and at the origin. This in turn puts an upper bound on the ratio of $\mathrm{G}$ today to it's minimum value, which, even in the most liberal case, is about $10^{37}$, and in much more realistic cases is more like 100 - vastly less than needed to solve the Penrose problem. The only way in which this scenario can be avoided is to very precisely define $F$ such that $G$ becomes equal to its current value very suddenly, requiring a degree of finetuning on at least the same order as the problem that Penrose identifies. We are therefore motivated to move away from looking for situations in which the field begins at small values and investigate the case of a large initial field.

\section{LARGE INITIAL FIELD}

An alternative way in which we can realize the dynamics we seek is to consider beginning the scalar field at high (positive) field values. We note that in such cases $F$ should be monotonically increasing towards high field values so that equation 1.2 obeys the second law of thermodynamics. To examine this possibility, consider the dynamics of a field that begins at rest at a high field value. From equation 2.10 we see that the acceleration of the field is proportional to $2 V \frac{F^{\prime}}{F}-V^{\prime}$. Thus we see that for this acceleration to be towards the minimum 
of the potential we need that asymptotically $F^{2} \leq V$, which in the case of our Higgs potential means that $F$ cannot asymptote to a function which grows faster than $\phi^{2}$.

As we have noted, to follow the second law of thermodynamics, we need $\dot{F}<0$ so that $G$, its inverse is increasing, and thus the black hole entropy will increase. In the case of a large initial field which we expect to roll back to the minimum of the Higgs potential, this means that $F$ cannot have local minima between the Higgs minimum and the initial value.

\section{A. Quadratic $F$}

The case in which $F \propto \phi^{2}$ is that examined in many cases of Higgs inflation (see e.g. Shaposhnikov [9]) and this term means the acceleration of the field tends to zero at large field values, giving rise to the slow-roll effect, as needed for inflation. This is usually established in the Einstein frame for convenience of calculation, but here we retain the Jordan frame as we will examine more general scenarios.

In recent work it was suggested that this alteration of the effective Newton constant could be responsible for 'turning off' gravity at high energy densities [3. Here we demonstrate why this limit is still singular in our case, and in section $\mathrm{V}$ we give a general argument as to why this cannot be achieved. The Hubble parameter is determined by equation 2.7, and thus grows at high field values. Since both kinetic and potential energy contribute to $H$, and both are positive, the Hubble parameter at a given value of $\phi$ is greater than the value it would take if the kinetic energy were zero:

$$
H>\sqrt{\frac{V}{F}}>V^{1 / 4} \propto \phi
$$

by the same consideration in the case of a Higgs potential. Thus we see from running our dynamics backwards that in such cases the cosmological model begins with an initial singularity $(H \rightarrow \infty$ at $\phi \rightarrow \infty)$. We shall show that this behaviour is universal in such models. The quadratic case is the fastest rate of growth of $F$ in which the field is driven to the minimum of its potential (see section V) but for our purposes the growth need not be quadratic. We thus further split the discussion into two cases; the first case to consider is where $F$ is unbounded from above (but grows slower than $\phi^{2}$ ), and the second is where $F$ asymptotes to a finite constant.

\section{B. If $F$ is unbounded above}

let us consider the following as an exemplar function:

$$
F=\frac{A^{2} \phi^{2}}{\left(\phi+\xi^{2}\right)}
$$

with $A, \xi$ constants, and $V$ is given by (3.1). We choose $\xi^{2}>>\Phi$, and could use the modulus of the numerator to make an even function if we wish to consider negative field values. In such a model we see the asymptotic behaviour required - $F$ becomes linear in $\phi$ at high field values, and is quadratic for $\phi<\xi^{2}$. We note that the condition for finding a non-trivial minimum was given in equation (2.11). Here we show that this cannot be realized:

$$
\frac{V^{\prime}}{V}-2 \frac{F^{\prime}}{F}=\frac{2 \xi^{2} \Phi^{2}+\Phi^{2} \phi+6 \xi^{2} \phi^{2}+7 \phi^{3}}{2 \phi\left(\xi^{2}+\phi\right)\left(\phi^{2}-\Phi^{2}\right)}
$$

which is never zero (or singular) away from $\phi=\Phi$. Therefore the complete dynamics of such a system closely matches those we want: the field begins at large field values where the effective Newton constant is almost zero, evolves through a phase in which it undergoes Higgs inflation, and eventually comes to rest at the minimum of the Higgs potential. The behaviour of this system is analysed in the Einstein frame in section $\mathrm{V}$, with the effective potential shown in figure 1 . Thus this model resembles the standard Higgs inflation case [9] and so can fit the Planck data [33. The current value of the Planck mass is (for $\xi^{2}>>\Phi$ ) given by $M_{p}=A \Phi / \xi$, whereas at high values of the scalar field (i.e. in the early universe) it tends towards $A \phi$, and hence tends to infinity $(G \rightarrow 0)$ at the initial singularity, thus removing the initial entropy problem.

Overall: this is a case that works. 


\section{F asymptotes to a finite constant}

In the second case we mirror the ideas of, for example, T-models or $\alpha$-attractors [23, 30] in which inflation is reproduced by a scalar field with a potential that tends towards a plateau at high field values. T-models, named in reference to their automobile counterparts for their apparent ubiquity, arises when the theory has a scalar field which has poles (usually two poles of order two) in its kinetic term. Such theories often arise as a result of supergravity theories. This is remedied through a field redefinition to recover a canonical kinetic term. In doing so the relationship between the original field, $\chi$, and its canonical counterpart, $x$, of necessity must monotonically map a finite range (the region between two poles, for example) in $\chi$ onto an infinite range in $x$, and hence $d \chi / d x \rightarrow 0$. Thus any potential $U(\chi)$ which is finite and has finite first derivative on this region will be rendered as having an infinite plateau in $x$. This occurs because

$$
\frac{d U}{d x}=\frac{d U}{d \chi} \frac{d \chi}{d x}
$$

with the latter term tending to zero. Thus these models are ubiquitous: Any well behaved potential in $\chi$ will render a plateau model suitable for generating inflation in the transformed field $x$.

In our case, we are not directly motivating our choice of function $F$ from a more fundamental theory which leads to this behaviour, but rather using such models as a qualitative exemplar of classes of functions which asymptote to constants. Since turning points for $F$ are disallowed, it should approach the plateau monotonically away from the Higgs minimum. Thus as the function increases, its derivative tends to zero from below.

In these situations slow roll inflation is achieved since the potential is sufficiently flat far from the origin, and finding the inflaton here should not be considered unnatural as there is always an infinity of parameter space with higher field values. As an example of this, we will choose the function

$$
F=M_{o}^{2}+A^{2} \tanh ^{2}(\phi / S),
$$

with $M_{0}, A$, and $S$ constants, where we shall choose $S$ to be considerably larger than the Higgs VEV. Note that there are a plethora of functions we could have used in this instance each of which although quantitatively distinct, we should expect to have qualitatively similar features. Our choice is motivated simply by the quadratic nature of the function about zero and the good approximation as such around the Higgs VEV, matching the usual Higgs inflation coupling, and its asymptotic plateau. In such a case, since the terms $F^{\prime 2} / F$ and $F^{\prime} V / F$ asymptote to zero, we see that in the large field limit we should expect to see the dynamics of the scalar field match those of general relativity, albeit with a significantly reduced Newton's constant. However, once the field exits the plateau phase its dynamics tend towards those of Higgs inflation (see e.g. [9]) which agree with the Planck observations 33 .

Let us note a few features of such choices of $F$. First we can show that the asymptotic behaviour of the system is indeed that the field comes to rest at the minimum of the Higgs potential. Again, the condition for a stationary point away from the Higgs minimum cannot be realized:

$$
\begin{aligned}
\frac{V^{\prime}}{V} & -2 \frac{F^{\prime}}{F}=\frac{\phi^{2}-\Phi^{2}}{4 \phi} \\
& -S \cosh ^{2}\left(\frac{\phi}{S}\right) \operatorname{coth}\left(\frac{\phi}{S}\right)\left(M_{o}(1+A) \tanh \left(\frac{\phi}{S}\right)\right)
\end{aligned}
$$

First we consider the case $\phi>0$. Finding a Laurent series for $V^{\prime} / V-2 F^{\prime} / F$ reveals that this can be expressed as a polynomial in $\phi$, the coefficients of each term being negative. Hence the sign of the function is fixed. Now since the total expression is odd, a parallel argument runs for $\phi<0$. Therefore the only possible points at which the field can be at rest are at the origin (which is an unstable equilibrium point, and thus would only hold if the field were always there) or at the minima of the Higgs potential (stable equilibria). Hence the field will eventually come to rest at the Higgs VEV. For $S>>\Phi$ we find that this gives an effective Planck mass of

$$
M_{p}^{2}=M_{o}^{2}\left(1+A^{2} \tanh ^{2}\left(\frac{\Phi}{S}\right)\right) \approx M_{o}^{2}
$$


whereas at high field values, the effective Planck mass is determined on the plateau of $F$ :

$$
M_{p}^{2} \approx M_{o}^{2}\left(1+A^{2}\right) \approx A^{2} M_{o}^{2}
$$

Thus we see that if we want to alleviate the initial fine-tuning problem we require that $A$ is of the order of $10^{123}$, thus reducing $G$, and hence the entropy count of primordial black holes, by this same factor.

In this bounded-above case, you do need a large number somewhere to make the potential high enough to overcome the $10^{123}$ factor in $G$. It does look like an unnaturalness in terms of having such a large number instead of one around unity; but a number even larger than this would still work, so it's not fine tuning in the sense of needing a rather specific value. Also on using a log prior [29] it's only tuned to around 1 part in 100 . If we in any case adopt the view that naturalness of such parameters is not a major issue [27, this case works too. However perhaps the unbounded-above case mentioned above is more appealing as you only need to introduce one new term $(\xi)$ which is bounded to be an order of magnitude above the Higgs minimum.

\section{FRAME TRANSFORMATION AND POTENTIAL}

Our analysis thus far has been carried out in the Jordan frame. However, if we want to make comparisons with inflation, it is often useful to transform to the Einstein frame. Here we will follow Kaiser (94), and note that by making a conformal transformation of the metric $\left(\hat{g}_{\mu \nu}=\Omega^{2} g_{\mu \nu}\right)$ we can rewrite the non-minimally coupled field as a scalar field with a transformed potential. The choice $\Omega^{2}=F / 6$ renders the action

$$
S=\int \sqrt{\hat{g}} \hat{R}+\frac{\partial_{\mu} \chi \partial^{\mu} \chi}{2}-U(\chi)
$$

wherein $\hat{R}$ is the Ricci scalar of the metric $\hat{g}$, and $\chi$ is obtained from solving

$$
\frac{d \chi}{d \phi}=\sqrt{\frac{F+3\left(F^{\prime}\right)^{2}}{4 F^{2}}}
$$

with the transformed potential

$$
U(\chi)=\frac{V(\phi)}{4 F^{2}}
$$

\section{A. Unbounded-above case}

Let us return our attention to the unbounded large field case where the function $F$ is that of 4.3): $F=\frac{A^{2} \phi^{2}}{\xi^{2}+\phi}$. To analyse the choices in the Einstein frame, we first note that the potential $U(\chi)$ can be expressed as

$$
U=\frac{V}{4 F^{2}}=\frac{\left(\Phi^{2}-\phi^{2}\right)^{2}}{4 A^{2} \phi^{4}}\left(\xi^{2}+\phi\right)^{2}
$$

and hence it becomes apparent that we should split our analysis into three regimes corresponding to (i) the initial large field, (ii) the region in which $A$ is most relevant, and (iii) the behaviour around the minimum of the Higgs potential: $\phi>>\xi, \Phi<\phi<<\xi$ and $\phi \approx \Phi$ respectively. In the first of these regimes we see that $F$ is essentially linear, and hence $\chi \propto \sqrt{x}$, and thus $U(\chi) \propto \chi^{4}$, and our field will begin its descent down the potential. In the latter two regimes $\chi \propto \sqrt{\phi}$ and hence at first $U(\chi) \approx \xi^{4}+\frac{2 \xi^{2}}{\chi^{2}}$, during which phase the potential is approximately a plateau, and the dynamics will match that of Higgs inflation, since $F \approx \frac{A^{2} \phi^{2}}{\xi^{2}}$ and thus our action would match that of Higgs inflation. In the final phase we can expand $U$ about $\Phi^{2}$, and we see that we once again recover the Higgs potential;

$$
U=\frac{\left(\Phi+\xi^{2}\right)^{2}}{4 A^{2} \Phi^{4}}\left(\chi-\Phi^{2}\right)^{2}+O\left(\chi-\Phi^{2}\right)^{3}
$$

Equation (5.5) refers to the unbounded-fromabove potential, but this time in the Einstein frame rather than the Jordan frame. This is an example that works. The point here is that you can see why, for example, $F>\phi^{2}$ doesn't work, and the nice plateau form of the potentials in this setup.

\section{B. What does not work}

Consider the case in which $F$ grows faster than $\phi^{2}$ for large field values. To make this explicit, consider $F \propto \phi^{n}$, where we shall later set 


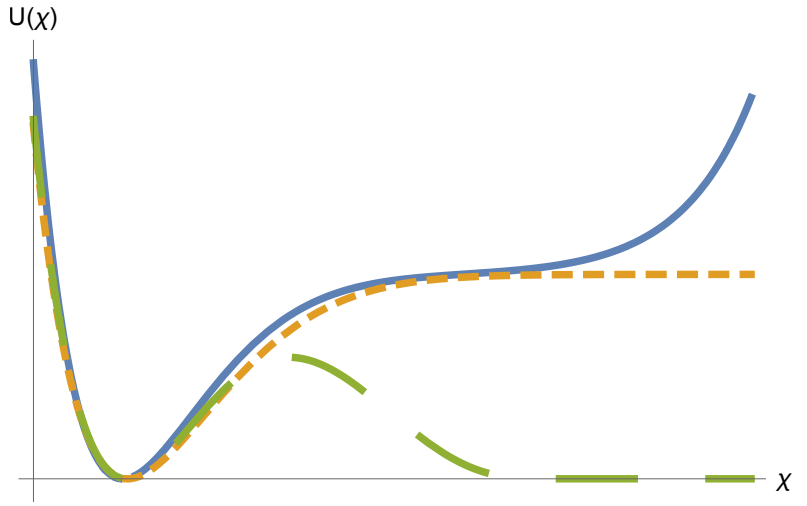

FIG. 1: Potential in $\chi$ for three different choices for $F$ corresponding to the usual quadratic (orange, short dashed), a quartic (green, long dashed) and $F=\frac{\phi^{2}}{A^{2}+\phi}$ (blue, solid). We see that in the latter two cases we recover the plateau for Higgs inflation near the minimum of the potential, and in the first case the field would roll off to infinity as described.

$n>2$. Then when we transform to the Einstein frame, we find

$$
\Omega^{2} \propto \phi^{n}, \quad \chi \propto \log \phi+O\left(\phi^{-2}\right)
$$

which together tells us that at large field values $\Omega^{2} \propto e^{\frac{2 \chi}{\sqrt{3}}}$ and thus we see that the transformed potential in the Einstein frame is given by

$$
U(\chi) \propto \frac{\left(\Phi^{2}-e^{\frac{4 \chi}{\sqrt{3} n}}\right)^{2}}{e^{\frac{4 \chi}{\sqrt{3}}}} \rightarrow e^{\frac{4 \chi}{\sqrt{3} n}(2-n)} .
$$

Hence if $n>2$ the potential is decreasing away from the origin, and the field will roll away to infinity in such cases. Thus our analysis in the Einstein frame matches that in the Jordan frame for these choices of high initial field values. In other words, if $F$ asymptotically grows faster than $\phi^{2}$ our cosmological solutions will push $\phi \rightarrow \infty$ forever, and we will recover neither inflation nor standard model physics. In fact this result only relies on the fact that $V$ must grow faster than $F^{2}$ to have a monotonically non-decreasing potential in $\chi$, since $\chi$ is by design a monotonic function of $\phi$. Together with the result of equation 4.1 which tells us that for generic potentials $V$ and functions $F, H \propto \sqrt{F U}$, we see that if both $F$ and $U$ are non-decreasing functions the only way to avoid a $H \rightarrow \infty$ singularity at large $\chi$ would be if both were bounded from above.
Let us consider that scenario. If we were to modify the Higgs potential such that it asymptotes to a constant we would still find the singularity unavoidable. Suppose $V=V_{\infty}$ and $F=F_{\infty}$ for $\phi>\phi_{m}$ say for some finite values of $V_{\infty}$ and $F_{\infty}$. Then the Klein-Gordon equation 2.10 reduces to a (somewhat rescaled) version of the usual inflaton dynamics on a flat potential:

$$
\ddot{\phi}+3 \dot{\phi} \sqrt{\frac{\dot{\phi}^{2}}{2 F_{\infty}}+\frac{V_{\infty}}{F_{\infty}}}=0
$$

and thus the kinetic energy of the inflaton tends to infinity, taking the Hubble parameter with it. Hence any large field model is necessarily singular, and if we require that $F$ is finite for all finite values of $\phi$ we see that one cannot achieve the goal of having the cosmological solution asymptote to one in which $G=0$ always [3] without either resorting to breaking the second law of thermodynamics or having to introduce a new principle by which the Higgs field could tunnel from infinity. The asymptotic behaviour of models in which $G$ is taken to be small whilst $\phi$ grows large differs qualitatively from that in which $G$ is set by hand to zero.

The approach of [3] requires $F \rightarrow \infty$ at the origin, so that does not work. The reason for this is that in this case, $V$ is still finite at the origin, and hence the Higgs particle becomes trapped there. To illustrate this we will work in the Einstein frame. Suppose $F \approx \phi^{n}$ for $n<0$. Then, close to $\phi=0$ we find $\chi \approx \sqrt{3} n / 2 \log (\phi)$. Thus $\phi \rightarrow 0$ as $\chi \rightarrow \infty$. There is ambiguity in taking the square root of $n^{2}$ in this calculation. However, taking a negative square root both changes the sign of the relationship between $\chi$ and $\phi$ and flips the potential about $\chi=0$ such that the complete dynamics is insensitive to this choice. The potential in the Einstein frame is rendered differently from that in equation 5.7:

$$
U(\chi)=\frac{V}{4 F^{2}} \rightarrow \Phi^{4} e^{-\frac{4 \chi}{\sqrt{3}}}
$$

This is decreasing away from the origin, hence the field would have to roll up the potential from infinity to overcome this. In terms of the original field (in the Jordan frame), this means that if $\phi$ begins at the origin it will remain there throughout the evolution. Thus we see that it is not 
possible that any such system (either beginning at large or small values of the field) can asymptotically become deSitter or Minkowski - it will always be singular.

\section{FURTHER STEPS}

We have shown there are a number of models that can provide the kind of desired unification described in Section 1 and also be compatible with the Planck observations. They are large field cases, with two options presented that work, see equations (4.2) and (4.5).

\section{A. Agreement with inflation}

The agreement of these models with inflationary observations are guaranteed because during the relevant phases of inflation the dynamics is well approximated by systems already analysed in the Encyclopedia Inflationaris [33]. One thing that should be considered carefully is the relationship between the Einstein and Jordan frames; the dynamics of inflation are usually analyzed in the Einstein frame since this where the scalar field is modelled as being minimally coupled to the (transformed) gravitational theory. However, other matter present will not have this coupling - in the Einstein frame this will appear as new couplings between the transformed field and the remaining matter. Therefore when considering reheating, for example, if one is to work in the Einstein frame, careful attention should be paid to these induced couplings.

Similarly the masses of the standard model particles are affected by the conformal transformation [24]. The precise way in which reheating is achieved in non-minimally coupled inflation is subject to a number of subtleties; there is a complex set of decays through $\mathrm{W}$ and $\mathrm{Z}$ bosons which in turn decay into fermions [8, 24]. The gauge bosons can back-react upon the Higgs condensate and thus the system can become highly nonperturbative, and the production of particles can be due to a force that appears 'spike-like' 21]. In order to fully model these situations, lattice simulations are used [36]. A full accounting of this is far outside the scope of this work, but fortunately, do not alter the our overall finding that Higgs inflation is viable. We have thus shown that both the question of what is the nature of the inflaton and why black holes do not dominate the entropy of the early universe can be reconciled in the same model.

\section{B. Can it be the Higgs?}

Throughout this paper we have used the Higgs field as the inflaton. The results we have obtained are not strictly dependent on the field in question being the Higgs itself, as any scalar field with the same non-minimal coupling to gravity and potential would give rise to an identical effect. However, we take the viewpoint that laboratory tested particle physics already has discovered a scalar field which is suitable for our purposes, and therefore is a strongly preferred candidate [20]. Therefore the question of interest is not whether any scalar field could be the inflaton, or answer questions about the low entropy of matter in the early universe. The answer to this has already been determined to be yes. The question we have asked is 'Could the Higgs field answer these questions?' What we have shown is that the answer is yes. This can be achieved without having to invoke new fields at all.

Using the Higgs field in this way provides interesting possibilities. Since the Higgs field is related to directly testable physics, the question arises as to whether there are observable consequences of the model for example in black hole physics, and particularly in terms of binary black hole coalescence accompanied by emission of gravitational radiation.

\section{Further issues}

Two issues regarding the 'turning off' of gravity arise. Firstly, in principle we need $1 / G$ to initially be infinity to completely turn gravity off. Even in this case, we note that there is a distinct qualitative difference in dynamics between models in which this is done through taking the 
limit of an unbounded from above field (as presented here) and formally setting $G=0$ in the equations of motion. In practice a finite value of $1 / G$ suffices to remove the entropy problem (and infinities are in any case to be avoided in real physics [19]). What that value is depends on which detailed model is employed. This needs further investigation, and is related to the second issue: in principle thermalisation can be achieved if the the Hubble parameter were very low to begin with, so there is time to smooth out the matter density in this weak gravity era. However in practice models do not exist where $H$ is very small initially. We therefore have to rely instead on the assumption that the universe starts off in a maximum entropy state, which will indeed be such a smooth state, instead of one chock full of black holes.

Finally, we need an investigation of black holes for such gravity theories: What is the di- rect relationship between $\mathrm{G}$ and entropy? What is Schwarzschild solution for these cases, the horizon location, and the Hawking temperature/entropy? A naive consideration of the Bekenstein-Hawking entropy 1.2 indicates that (keeping matter fixed) the relationship is linear. We note that since the Schwarzschild solution is vacuum solution, it is a solution of the nonminimally coupled theory for finite $G$. However the event horizon is defined topologically as the past horizon of future null infinity - this needs modification in case in which $G$ can vary over time, and a more detailed analysis should be based on a more local concept defined by a dynamical horizon in this context [4]. We leave these issues for separate discussion.

We are grateful to the anonymous referee whose insightful comments have greatly improved this article.
1] Ade, P et al (2016). "Planck 2015 results-xiii. cosmological parameters" Astronomy and Astrophysics 594 : A13.

[2] Aghanim, N et al (2018) "Planck 2018 results. VI. Cosmological parameters" arXiv preprint arXiv:1807.06209.

[3] Alexander, S, Barrow, J D, and Magueijo, J (2016). Turning on gravity with the Higgs mechanism. CQG 33: 14LT01.

[4] Ashtekar, A and Krishnan, B (2003) "Dynamical Horizons and their Properties" Phys Rev. D66: 104030

[5] Barbon, Casas, J., Elias-Miro, J. and Espinosa, J. (2015) "Higgs Inflation as a Mirage" JHEP 09027

[6] Barbon, J and Espinosa J (2009) "On the Naturalness of Higgs Inflation" Phys.Rev. D79 081302

[7] Bezrukov, F (2013) "The Higgs field as an inflaton" Classical and Quantum Gravity 30:214001

[8] Bezrukov, F, Gorbunov, D and Shaposhnikov, M (2009) "On initial conditions for the Hot Big Bang" JCAP 0906029

[9] Bezrukov, F, Magnin, A, Shaposhnikov, M, and Sibiryakov, S (2011) "Higgs inflation: consistency and generalisations" JHEP 1101016

[10] Bezrukov, F and Shaposhnikov, M (2008) "The Standard Model Higgs boson as the inflaton" Physics Letters B 659:703 16 .
[11] Brax, P and Davis, A C (2014) "Conformal inflation coupled to matter." JCAP 2014:019.

[12] Burgess C P, Lee H M, and Trott M, (2009) "Power-counting and the Validity of the Classical Approximation During Inflation" JHEP 0909103

[13] Burgess C P, Lee H M, and Trott M, (2010) "On Higgs Inflation and Naturalness" JHEP 1007 007

[14] Clough, K, Flauger, R, and Lim, E A (2018) "Robustness of inflation to large tensor perturbations" JACP 2018(05), 065.

[15] Clough, K, Lim, E A, DiNunno, B S, Fischler, W, Flauger, R, and Paban, S (2017). "Robustness of inflation to inhomogeneous initial conditions" JCAP 2017(09), 025.

[16] East, W E, Kleban, M, Linde, A, and Senatore, L (2016) "Beginning inflation in an inhomogeneous universe" JCAP 2016(09), 010.

[17] Ellis G F R, Maartens R, and MacCallum M A H (2012) Relativistic Cosmology (Cambridge: Cambridge University Press)

[18] Ellis, G and Madsen, M, (1991) "Exact scalar field cosmologies" Class. Quant. Grav 84

[19] Ellis, G F, Meissner, K A, and Nicolai, H (2018). "The physics of infinity" Nature Physics 14:770.

[20] Ellis G and Uzan J P (2014) "Inflation and the Higgs particle" Astronomy and Geophysics 55:1-19. 
[21] Ema, Y, Jinno, R, Mukaida, K and Nakayama, K (2017) "Violent Preheating in Inflation with Nonminimal Coupling" JCAP 1702045

[22] Ferreira, P G, Hill C T, Noller J, and Ross G G (2018) "Inflation in a scale-invariant universe." Physical Review D 97: 123516.

[23] Galante, M, Kallosh, R, Linde A, and Roest, D (2015) "The Unity of Cosmological Attractors" Physical Review Letters 114:141302

[24] Garcia-Bellido, J, Figueroa, D and Rubio, J (2009) "Preheating in the Standard Model with the Higgs-Inflaton coupled to gravity" Phys.Rev. D79 063531

[25] Garcia-Bellido, J, Rubio, J, Shaposhnikov, M, and Zenhäusern, D (2011) "Higgs-dilaton cosmology: from the early to the late universe" Physical Review D 84: 123504.

[26] Greene, B, Hinterbichler, K, Judes, S, and Parikh, M (2011) "Smooth initial conditions from weak gravity" Physics Letters B 697: 178183.

[27] Hossenfelder, S (2018) Lost in Math: How beauty leads physics astray (Basic Books).

[28] Ijjas A and Steinhardt P J (2016) "Implications of Planck 2015 for inflationary, ekpyrotic and anamorphic bouncing cosmologies" Class. Quant. Grav.33: 044001 [arXiv 1512.09010].

[29] Jeffreys, H (1946). "An Invariant Form for the Prior Probability in Estimation Problems" Proc Ro Soc A 186: 453461

[30] Karananas, G, and Rubio, J (2016) "On the geometrical interpretation of scale-invariant models of inflation" Physics Letters B 761:223

[31] Kleban, M, and Senatore, L (2016) "Inhomogeneous anisotropic cosmology" JCAP 2016:022.

[32] Lerner, R and McDonald, J (2010) "Higgs Inflation and Naturalness" JCAP 1004015

[33] Martin, J, Ringeval, C, and Vennin, V (2014) "Encyclopdia inflationaris" Physics of the Dark Universe 5:75-235. arXiv:1303.3787

[34] Munoz, J and Kamionkowski, M (2015) "Equation-of-State Parameter for Reheating" Phys.Rev. D91: 043521
[35] Penrose, R (2016) Fashion, Faith, and Fantasy in the New Physics of the Universe (Princeton: Princeton University Press)

[36] Repond, J and Rubio, J (2016) "Combined Preheating on the lattice with applications to Higgs inflation" JCAP 1607043

[37] Rubio, J (2018) "Higgs inflation" arXiv: 1807.02376

[38] Starobinsky, A (1980) "A new type of isotropic cosmological models without singularity" Physics Letters B 9199

[39] Synge, J L (1955) Relativity: the General Theory (Amsterdam: North Holland)

[40] Vachaspati T and Trodden M (1999) "Causality and cosmic inflation" Phys. Rev. D61:023502

[41] Weinberg S W Gravitation and Cosmology

\section{Appendix A: the cosmological equations}

The cosmological equations are, the Friedmann Equation:

$$
F\left(H^{2}+\frac{F^{\prime}}{F} H \dot{\phi}+\frac{k}{a^{2}}\right)=\rho,
$$

the Raychaudhuri (acceleration) equation:

$2 F\left(\dot{H}+H^{2}\right)=-(\rho+3 P)-F^{\prime} \dot{\phi} H-F^{\prime \prime} \dot{\phi}^{2}-F^{\prime 2} \ddot{\phi}$,

and the Klein-Gordon Equation:

$$
\begin{array}{r}
\left(1+\frac{F^{\prime}}{2 F}\right)(\ddot{\phi}+3 H \dot{\phi})+V^{\prime} \\
=-\frac{F^{\prime}}{F}\left(2 V+\frac{\rho_{m}-3 P_{m}}{2}+\left(1-F^{\prime \prime}\right) \frac{\dot{\phi}^{2}}{2}\right)
\end{array}
$$

The latter is equivalent to the conservation equation for the scalar field, which in turn guarantees the consistency of A1 and A2. 